\title{
Asymmetry of critical closing pressure following head injury
}

\author{
A Kumar, E A Schmidt, M Hiler, P Smielewski, J D Pickard, M Czosnyka
}

J Neurol Neurosurg Psychiatry 2005;76:1570-1573. doi: 10.1136/jnnp.2004.059493

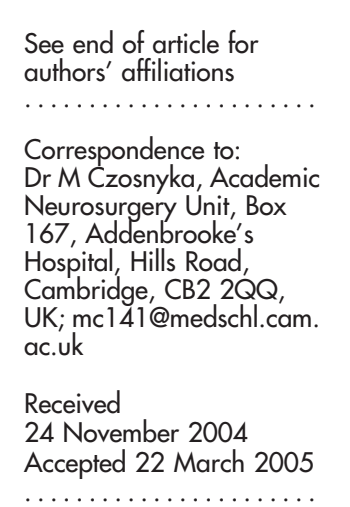

\begin{abstract}
Objective: Critical closing pressure (CCP) is the arterial pressure below which the vessels collapse. Hypothetically it is the sum of intracranial pressure (ICP) and vessel wall tension in the cerebral circulation. This study investigated transhemispherical asymmetry of CCP by studying its correlation with radiological findings on computed tomography (CT) scans in head injury patients.

Method: ICP, arterial blood pressure, and middle cerebral artery blood flow velocity were recorded daily in 119 ventilated patients. Waveforms were processed to calculate CCP. CT scans were analysed according to a system based on the Marshall classification.

Results: Left-right differences in CCP correlated with midline shift on the CT scan $(r=0.48 ; p<0.02)$. Asymmetry of CCP also corresponded with the side of the head lesion $(p<0.007)$ and the side of the craniotomy where it was performed $(p<0.006)$. Absolute CCP weakly correlated with brain swelling $(r=-0.23 ; p<0.03)$ and arterial pressure $(r=0.21 ; p<0.02)$ but did not correlate with ICP. Cerebral perfusion pressure calculated as the difference between mean arterial pressure and CCP did not correlate with outcome, but "traditional" cerebral perfusion pressure (mean arterial pressure minus intracranial pressure) did.

Conclusions: Critical closing pressure is disturbed by localised brain lesions. Its asymmetry corresponds to asymmetrical findings on CT scans. CCP seems to describe vascular resistance better than ICP.
\end{abstract}

$T$ he critical closing pressure (CCP) of an artery is defined as the intraluminal pressure below which the artery collapses and flow ceases within that vessel. This concept was first formulated by Burton in 1951. ${ }^{1}$ CCP for cerebral arteries represents the cumulative effect of the intracranial pressure (ICP) and the tension of arterial walls. ${ }^{2}$ A method for calculating CCP using transcranial Doppler ultrasonography has been proposed by Aaslid ${ }^{3}$ so that CCP is becoming a physiological index rather than just a theoretical entity. It is specially attractive as it can be assessed purely noninvasively, and a number of neuroscientists have recently studied CCP in various scenarios. ${ }^{4-6}$

The major determinants of outcome after serious head injury include systemic hypotension and intracranial hypertension. The cerebral perfusion pressure is reduced in either case and may result in a decrease in cerebral blood flow rendering the brain susceptible to secondary ischaemic insults. Therefore, the key to management is a cerebral perfusion pressure orientated strategy. ${ }^{7}$ When applied to the cerebral circulation, CCP may characterise the downstream resistance of the cerebral vascular bed more accurately than ICP alone and perhaps should be included in the estimation of cerebral perfusion pressure. ${ }^{8}$ This interesting hypothesis requires verification in clinical conditions.

With bilateral transcranial Doppler ultrasonography, CCP can be assessed independently for both hemispheres. Very little has been demonstrated with regard to the symmetry of CCP, with the exception of documented asymmetry in CCP and its correlation with asymmetry of vessel narrowing in patients with carotid artery stenotic disease. ${ }^{5}$ We investigated how the CCP is affected in patients with head injuries, paying particular interest to asymmetrical CCP patterns between the cerebral hemispheres and its relation to hemisphere swelling assessed by head computed tomography (CT) scanning.

\section{PATIENTS AND METHODS}

\section{Patient population}

A total of 119 patients (19 women, 100 men; mean age 36 years, range 16-76) with head injuries were included in this study. The median initial Glasgow Coma Score was 7 (range 3-15). All patients were sedated, paralysed, and ventilated. They were treated in accordance with the neurocritical care unit protocol to optimise cerebral perfusion pressure. ${ }^{9}$ The patients were kept normothermic, ICP was maintained with boluses of mannitol, and arterial blood pressure (ABP) was maintained by volume expansion and dopamine.

\section{Monitoring and data analysis}

We measured the ABP directly from the radial artery and the ICP with a fibreoptic transducer inserted intraparenchymally into the right frontal region. We calculated the cerebral perfusion pressure as the difference between the mean $\mathrm{ABP}$ and mean ICP. In addition, we measured the cerebral blood flow velocities by the daily insonation of both the left and right middle cerebral artery for 20 minutes to two hours. The duration of recording conducted on the patients ranged from one to 11 days (median 3 days). Transcranial Doppler, ICP, and ABP measurements are conducted routinely for head injured patients. ${ }^{10}$ We retrospectively analysed routine clinical and brain monitoring data, prospectively collected and approved by the local multidisciplinary Neuro Critical Care Users group. No such data with which individuals could have been identified were included. At the time of data collection, the local ethical committee approval was not required. We assessed outcome using Glasgow Outcome Scale at six months after injury.

The analogue outputs from the transcranial Doppler unit and the pressure devices were fed into an analogue digital converter connected to a laptop computer. We calculated time-averaged values of $\mathrm{ICP}, \mathrm{ABP}$, and cerebral perfusion pressure from the saved data by integrating waveforms over five seconds. After removing artefacts, time-averaged values over the same five second periods were also calculated for the flow velocities (FV). The CCP was calculated according to formula ${ }^{6}$ :

Abbreviations: $A B P$, arterial blood pressure; $C C P$, critical closing pressure; ICP, intracranial pressure; FV, flow velocity 
$\mathrm{CCP}=\mathrm{ABP}_{\text {MEAN }}-$ (first harmonic of $\mathrm{ABP}$ pulse/first harmonic of $\mathrm{FV}$ pulse $)^{\star} \mathrm{FV}_{\text {MEAN }}$

We averaged the measured variables over the total time of monitoring so that every patient was represented by one set of results: left CCP, right CCP, the difference between left and right CCP, ICP, ABP, flow velocities for the left and right sides of the circulation, and cerebral perfusion pressure. Furthermore, CT findings were included in this data set and consisted of the extent of brain swelling, midline shift, and the side of the lesion. We estimated the cerebrovascular resistance as the ratio of cerebral perfusion pressure and mean flow velocity.

\section{CT analysis}

We selected the CT scans of the head injured patients that were closest to the date of transcranial Doppler recording for analysis and graded the abnormalities according to a simplified version ${ }^{11}$ of the Marshall classification. ${ }^{12}$ The extent of brain swelling was determined by evaluating the effacement of ventricles, disappearance of sulci, basal cistern compression, and a reduction in the delineation between grey and white matter. Based on the number of the abovementioned features displayed in the scan, a score out of four $(0=$ no swelling, $4=$ severe swelling $)$ was assigned to each patient. We recorded the side of the major lesion as well as any midline shift.

\section{Statistical analysis}

Data in table 2 are presented as mean (SD). We analysed the correlation between different variables using linear regression and the calculation of Pearson's $r(\alpha$ level $<0.05)$. Other statistical analyses were performed using the analysis of variance test. All other tests used are specified in the results.

\section{RESULTS}

Table 1 gives the location of the head injury lesions in the patients included in the study. There was a midline shift in 32 of the 119 cases $(27 \%)$. The shift was from left to right in 20 patients and right to left in 12. Table 2 lists the mean (SD) of pressure, flow, and derived haemodynamic parameters.

The results show a significant correlation between differences in CCP across hemispheres and midline shift $(r=0.48$; $\mathrm{p}<0.02$ ), indicating that a lower CCP can be seen at the side from which brain expands (fig 1). A similar picture was seen

Table 1 Side of lesion on computed tomography scans

\begin{tabular}{ll}
\hline Lesion type & No (\%) \\
\hline Right & $44(36)$ \\
Left & $38(32)$ \\
Bilateral & $12(10)$ \\
None & $26(22)$ \\
\hline
\end{tabular}

Table 2 Averaged haemodynamic indices

\begin{tabular}{ll}
\hline Index & Mean (SD) \\
\hline Arterial blood pressure $(\mathrm{mm} \mathrm{Hg})$ & $96.0(9.5)$ \\
Intracranial pressure $(\mathrm{mm} \mathrm{Hg})$ & $17.5(7.3)$ \\
Cerebral perfusion pressure $(\mathrm{mm} \mathrm{Hg})$ & $78.7(8.9)$ \\
Blood flow velocity, left $(\mathrm{cm} / \mathrm{s})$ & $58.7(24.7)$ \\
Blood flow velocity, right $(\mathrm{cm} / \mathrm{s})$ & $58.5(26.2)$ \\
Critical closing pressure, left & $19.29(19.92)$ \\
Critical closing pressure, right & $20.86(19.73)$ \\
\hline
\end{tabular}

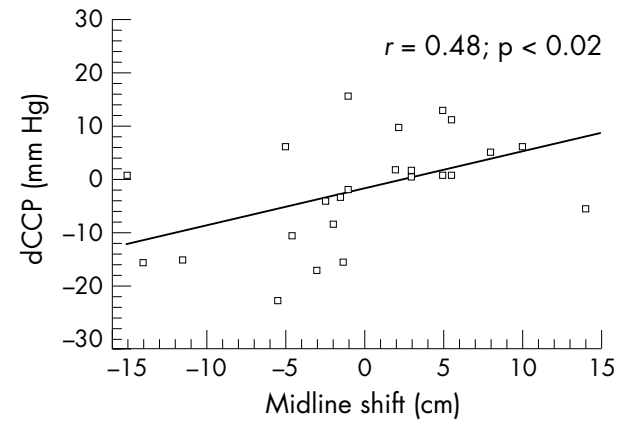

Figure 1 Significant correlation between left-right difference in critical closing pressure (dCPP) and left-right midline shift (measured in $\mathrm{cm}$ from the CT scan). The relation indicates that on the side of brain expansion $\mathrm{CCP}$ is lower.

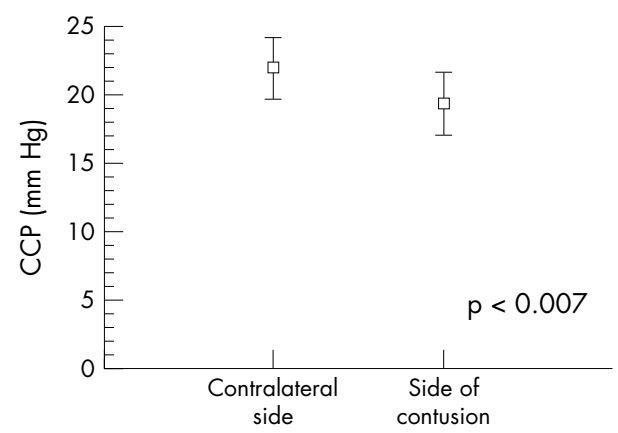

Figure 2 Difference between critical closing pressure (CPP) (means and $95 \%$ confidence bars) in contused hemisphere and uninjured hemisphere. CCP is lower at the side of contusion (paired signed rank test).

in patients with unilateral dominant contusion. CCP was lower at the side of contusion $(\mathrm{p}<0.007$; signed rank test) (fig 2). In patients with one-side craniotomy $(n=16)$ CCP was lower at the side of surgery $(\mathrm{p}<0.006$; signed rank test $)$ (fig 3). CCP was not correlated with measured ICP ( $p=0.17)$. The correlation between CCP and estimated cerebrovascular resistance was quite strong $(r=0.50 ; \mathrm{p}<0.0001)$ (fig 4), indicating greater CCP associated with greater vascular resistance. CCP correlated inversely with brain swelling $(r=-0.23 ; \mathrm{p}<0.03)$. CCP did not correlate with outcome at six months after injury. In those individual cases where time related changes in ICP were recorded, good agreement can, anecdotally, be seen between dynamics of ICP and CCP (fig 5).

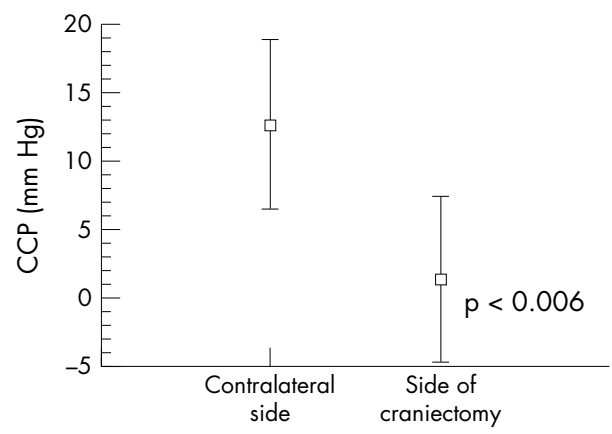

Figure 3 Differences in critical closing pressure (CCP) in 16 patients with one side craniotomy (mean values and 95\% confidence bars). Analysis shows that that CCP is lower at the side of craniotomy (paired signed rank test). 


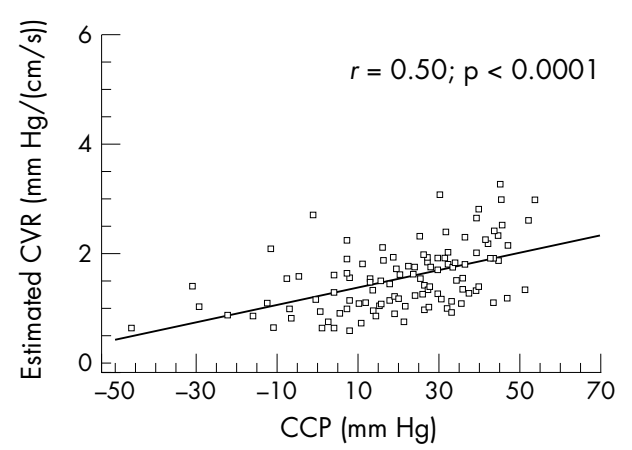

Figure 4 Relationship between CCP (averaged on left and right side) and estimator of cerebrovascular resistance (CVR). The correlation is highly significant.

To investigate what is the better measure for cerebral perfusion pressure: difference between $\mathrm{ABP}$ and ICP or difference between $\mathrm{ABP}$ and $\mathrm{CCP}$, the analysis of outcome seems to be helpful, as greater mortality was historically always correlated with lower perfusion pressure. In those patients who died, "traditional" CPP (ABP-ICP) was significantly lower than in those who survived (died: 73.5 (14) mm Hg; survived: 79.5 (8.1) mm Hg; p<0.03; MannWhitney test). Correspondingly, the difference in ABP-CCP in these two groups of patients was not significant (died: 77 (26) $\mathrm{mm} \mathrm{Hg}$; survived 74 (35) $\mathrm{mm} \mathrm{Hg}$ ).

\section{DISCUSSION}

In the present study we sought to determine how CCP was affected by one-sided head lesions and to what extent did changes in CCP reflect findings on CT scans. The results show that CCP was asymmetrical across the hemispheres. More than $15 \%$ of our patients had an absolute difference in leftright CCP greater than the $95 \%$ confidence limit evaluated in healthy volunteers $\left(14 \mathrm{~mm} \mathrm{Hg}^{13}\right)$. In our material, the asymmetry of CCP was significantly correlated with midline shift, side of the original lesion, and side of the craniotomy. We found that CCP was lower on the side of the predominant hemisphere swelling and lower on both the side of the contusion and the craniotomy.

Absolute CCP had a significant (although weak) correlation with the extent of brain swelling, with a greater degree of swelling corresponding to a lower CCP. Conceptually, CCP has been compared to the sum of ICP and the tension of

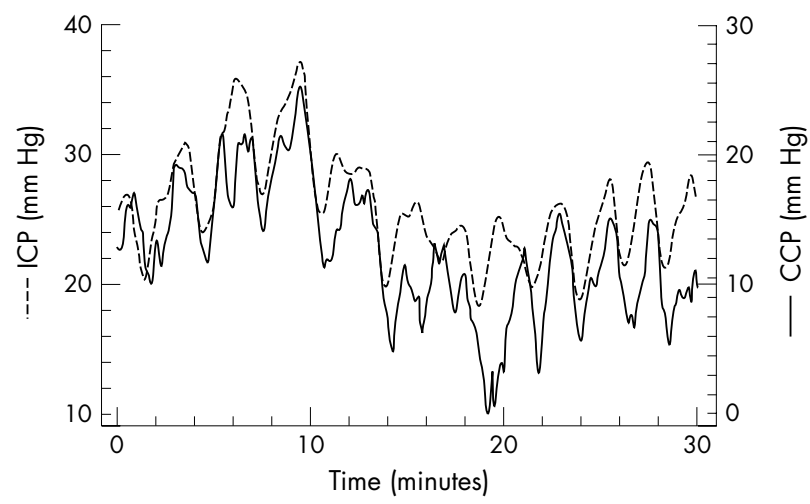

Figure 5 Example of high dynamics of intracranial pressure (ICP) recorded during 30 minutes of TCD examination. ICP changed from 35 to $17 \mathrm{~mm} \mathrm{Hg}$ and exhibited pattern of regular slow vasogenic waves of the period of around 2.5 minutes. Although the mean value of critical closing pressure (CCP) was strikingly different than mean value of ICP, changes in the CCP waveform did at times match the changes in ICP. arterial walls. ${ }^{2}$ However in the relatively large group of the patients in the present study, the correlation between mean CCP and ICP was not significant. This, together with a lower CCP on the side of brain contusion or brain expansion in cases with visible midline shift, questions the role of CCP as a useful estimator of ICP.

On the other hand, if the cerebrovascular resistance can be simplistically expressed as the ratio: CPP/FV, CCP appears to correlate well with cerebrovascular resistance. However, the correlation may be artificial and result from the mathematical formulae rather than physiological associations. Furthermore, having CCP related to tone and therefore vascular resistance corresponds better to the CT findings than if it were related to ICP. The ICP will be higher on the side of the brain that expands in response to trauma as well as the side of the head that has sustained the dominant contusion and the therapeutic craniotomy. However, if the CCP is linked to the ICP, we would expect a raised CCP in those hemispheres that have sustained the injuries. Our results show that CCP was lower in these regions and that this difference may be due to damaged and dilated vessels which would have a lower vascular resistance than the vessels in the uninjured hemisphere.

Our results also cast a shadow on the interesting thesis that cerebral perfusion pressure can be expressed better by the difference between mean arterial pressure and CCP rather than by the difference of mean arterial pressure and ICP. ${ }^{8}$ CCP is undoubtedly an interesting variable with many possible clinical applications, ${ }^{14}$ however, the fact that vascular abnormalities (like vasospasm ${ }^{15}$ ) may alter its original interpretation, ${ }^{2}$ is however disturbing. In our material, out of 119 patients, 16 had negative CCP without clear reason. Therefore, this index should be used with due care in clinical applications.

\section{CONCLUSION}

Critical closing pressure is a haemodynamic index that is affected in head injury patients. Side-to-side difference in CCP correlates with asymmetry of the injured brain. CCP is a poor estimator of mean intracranial pressure; it better describes cerebral vascular tone.

\section{ACKNOWLEDGEMENTS}

The authors are in debt to all members of the team participating in data collection: Mrs P Al-Rawi, Mrs C Turner, Mrs M Balestreri, Dr S Piechnik, Dr L Steiner, and all nursing and research staff of NCCU.

\section{Authors' affiliations}

A Kumar, University of Cambridge Clinical School, Addenbrooke's Hospital, Cambridge, UK

E A Schmidt, M Hiler, P Smielewski, J D Pickard, M Czosnyka, Academic Neurosurgery Unit, Addenbrooke's Hospital, Cambridge UK Supported by MRC Programme Grant (MRC G9439390 ID 56833)

Competing interests: none declared

M Czosnyka is on leave from Warsaw University of Technology, Poland.

\section{REFERENCES}

1 Burton AC. On the physical equilibrium of the small blood vessels. Am J Physiol $1951 ; 164: 319-29$

2 Dewey RC, Pieper HP, Hunt WE. Experimental cerebral hemodynamics. Vasomotor tone, critical closing pressure, and vascular bed resistance. Neurosurgery 1974;41:597-606.

3 Aaslid R. Cerebral haemodynamics. In: Newel DW, Aaslid R, eds. Transcranial Doppler. New York: Raven Press, 1992:49-55.

4 Carey BJ, Eames PJ, Panerai RB, et al. Carbon dioxide, critical closing pressure and cerebral haemodynamics prior to vasovagal syncope in humans. Clin Sci 2001;101:351-8.

5 Gooskens I, Schmidt EA, Czosnyka M, et al. Pressure-autoregulation, CO reactivity and asymmetry of haemodynamic parameters in patients with carotid artery stenotic disease. A clinical appraisal. Acta Neurochir (Wien) 2003; 145:527-32 
6 Aaslid R, Lash SR, Bardy GH, et al. Dynamic pressure-flow velocity relationships in the human cerebral circulation. Stroke 2003;34:1645-9.

7 Rosner MJ, Daughton S. Cerebral perfusion pressure management in head injury. J Trauma 1990;30:933-41.

8 Weyland A, Buhre W, Grand S, et al. Cerebrovascular tone rather than intracranial pressure determines the effective downstream pressure of the cerebral circulation in the absence of intracranial hypertension. J Neurosurg Anesthesiol 2000;12:210-16.

9 Patel HC, Menon DK, Tebbs S, et al. Specialist neurocritical care and outcome from head injury. Intensive Care Med 2002;28:547-53.

10 Czosnyka M, Smielewski P, Piechnik S, et al. Critical closing pressure in cerebrovascular circulation. J Neurol Neurosurg Psychiatry 1999;66:606-11
11 Schmidt EA, Czosnyka M, Steiner LA, et al. Asymmetry of pressure autoregulation after traumatic brain injury. J Neurosurg 2003;99:991-8.

12 Marshall L, Marshall S, Klauber MR, et al. A new classification of head injury based on computerised tomography. J Neurosurg 1991;75(suppl):S14-S21.

13 Schmidt EA, Piechnik SK, Smielewski P, et al. Symmetry of cerebral hemodynamic indices derived from bilateral transcranial Doppler. J Neuroimaging 2003;13:248-54.

14 Panerai RB. The critical closing pressure of the cerebral circulation. Med Eng Phys 2003;25:621-32.

15 Soehle M, Czosnyka M, Pickard JD, et al. Critical closing pressure in subarachnoid hemorrhage: effect of cerebral vasospasm and limitations of a transcranial Doppler-derived estimation. Stroke 2004;35:1393-8.

\section{Clinical Evidence-Call for contributors}

Clinical Evidence is a regularly updated evidence-based journal available worldwide both as a paper version and on the internet. Clinical Evidence needs to recruit a number of new contributors. Contributors are healthcare professionals or epidemiologists with experience in evidence-based medicine and the ability to write in a concise and structured way.

Areas for which we are currently seeking authors:

- Child health: nocturnal enuresis

- Eye disorders: bacterial conjunctivitis

- Male health: prostate cancer (metastatic)

- Women's health: pre-menstrual syndrome; pyelonephritis in non-pregnant women

However, we are always looking for others, so do not let this list discourage you.

\section{Being a contributor involves:}

- Selecting from a validated, screened search (performed by in-house Information Specialists) epidemiologically sound studies for inclusion.

- Documenting your decisions about which studies to include on an inclusion and exclusion form, which we keep on file.

- Writing the text to a highly structured template (about 1500-3000 words), using evidence from the final studies chosen, within 8-10 weeks of receiving the literature search.

- Working with Clinical Evidence editors to ensure that the final text meets epidemiological and style standards.

- Updating the text every six months using any new, sound evidence that becomes available. The Clinical Evidence in-house team will conduct the searches for contributors; your task is simply to filter out high quality studies and incorporate them in the existing text.

- To expand the topic to include a new question about once every 12-18 months.

If you would like to become a contributor for Clinical Evidence or require more information about what this involves please send your contact details and a copy of your CV, clearly stating the clinical area you are interested in, to Klara Brunnhuber (kbrunnhuber@ bmigroup.com).

\section{Call for peer reviewers}

Clinical Evidence also needs to recruit a number of new peer reviewers specifically with an interest in the clinical areas stated above, and also others related to general practice. Peer reviewers are healthcare professionals or epidemiologists with experience in evidence-based medicine. As a peer reviewer you would be asked for your views on the clinical relevance, validity, and accessibility of specific topics within the journal, and their usefulness to the intended audience (international generalists and healthcare professionals, possibly with limited statistical knowledge). Topics are usually 1500-3000 words in length and we would ask you to review between 2-5 topics per year. The peer review process takes place throughout the year, and our turnaround time for each review is ideally 10-14 days.

If you are interested in becoming a peer reviewer for Clinical Evidence, please complete the peer review questionnaire at www.clinicalevidence.com or contact Klara Brunnhuber (kbrunnhuber@bmigroup.com). 